\title{
Correlation between Methylation and Acetylation within the Cell due to Environmental Factors
}

\author{
Tiffany A. Pompa, William L’Amoreaux and Chang Hui Shen
}

\section{Department of Biology, College of Staten Island, Staten Island, New York 10314}

The inner works of the genome involve the integration of various processes within the cell, such as DNA methylation, chromatin remodeling and histone modification. These components allow the cell to maintain its stability with different chromatin conformations that ensure proper genetic expression. The genome of an organism is constructed in such a way that its expression can be correlated with the effects of the outside environment. Functions within a cell are uniform but become heterogeneous when outside factors are introduced and genes are expressed.

Using immunohistochemical analysis, the effects of environmental stress on the epigenetic state of the genome is observed through phenotypic and genetic expression. It was determined that a correlation between methylation and acetylation of the histones is caused by environmental stimuli. Wild-type strains of the yeast, sacchromyces cerevesiae, were employed to study genetic reaction to outside components such as copper. Copper causes the induction of the CUPI gene which creates activity within the cell, thus acetylation occurs and methylation ceases.

A continuation in outside factors affecting reactions within the genome will be studied along with the stimulus of hydroxyurea and ultra-violet light. This study should reveal molecular mechanisms used by the cell to sustain unsuitable conditions.

References:

[1] R. Jaenisch, A. Bird. Nature Genetics 33 (2003): 245. 

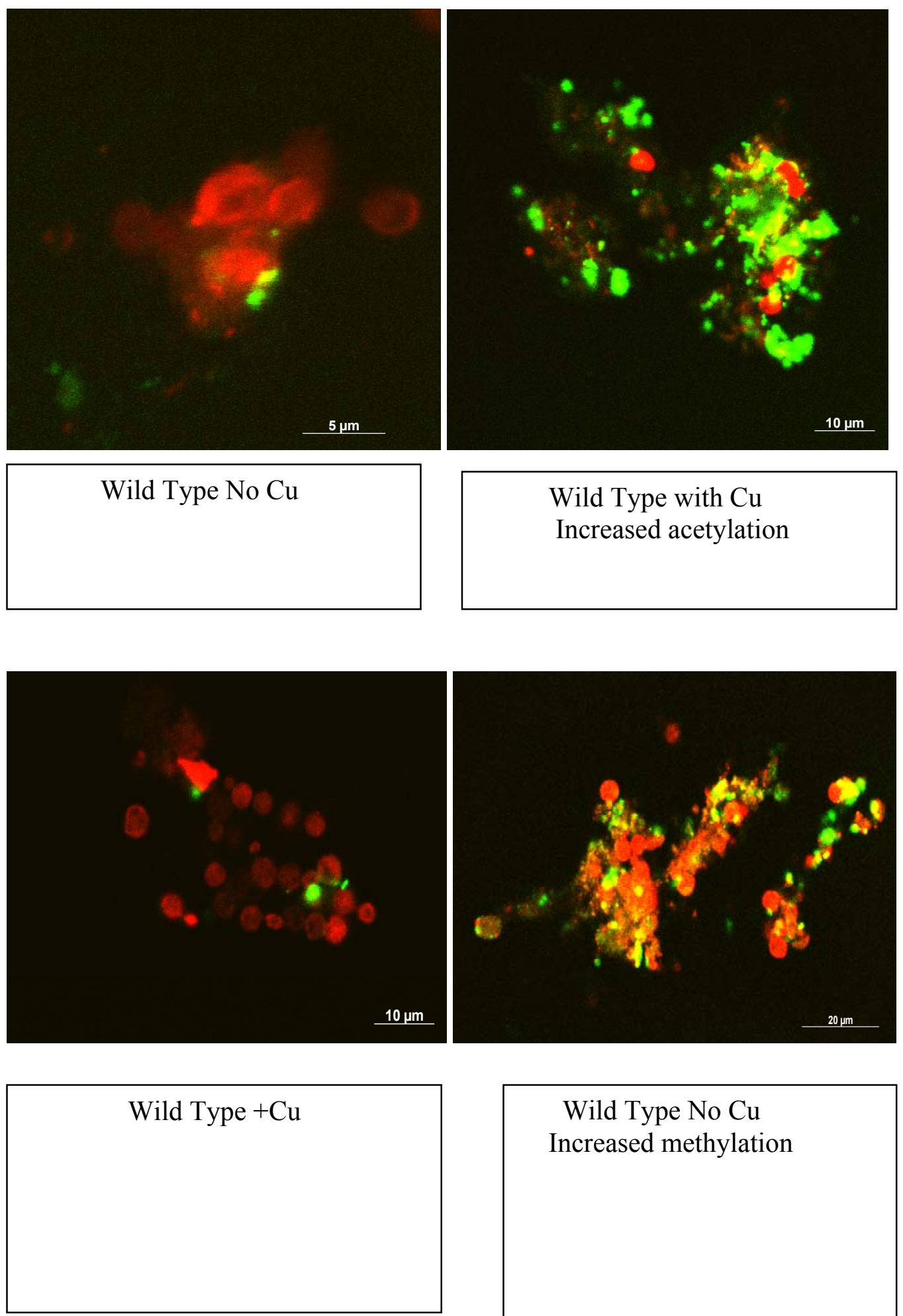

Wild Type No $\mathrm{Cu}$ Increased methylation 